\title{
UCRL-TR-206313
}

\section{Simulating Afterburn with LLNL Hydrocodes}

Lara D. Daily

August 31, 2004 
This document was prepared as an account of work sponsored by an agency of the United States Government. Neither the United States Government nor the University of California nor any of their employees, makes any warranty, express or implied, or assumes any legal liability or responsibility for the accuracy, completeness, or usefulness of any information, apparatus, product, or process disclosed, or represents that its use would not infringe privately owned rights. Reference herein to any specific commercial product, process, or service by trade name, trademark, manufacturer, or otherwise, does not necessarily constitute or imply its endorsement, recommendation, or favoring by the United States Government or the University of California. The views and opinions of authors expressed herein do not necessarily state or reflect those of the United States Government or the University of California, and shall not be used for advertising or product endorsement purposes.

This work was performed under the auspices of the U.S. Department of Energy by University of California, Lawrence Livermore National Laboratory under Contract W-7405-Eng-48. 


\title{
Simulating AfTerburn With LLNL HydrocodeS
}

\author{
Lara D. Daily \\ Defense Systems Analysis Group, Engineering \\ Q-Division, Nonproliferation Arms Control and International Security \\ Lawrence Livermore National Laboratory \\ Livermore, CA 94551
}

\begin{abstract}
Presented here is a working methodology for adapting a Lawrence Livermore National Laboratory (LLNL) developed hydrocode, ALE3D, to simulate weapon damage effects when afterburn is a consideration in the blast propagation. Experiments have shown that afterburn is of great consequence in enclosed environments (i.e. bomb in tunnel scenario, penetrating conventional munition in a bunker, or satchel charge placed in a deep underground facility). This empirical energy deposition methodology simulates the anticipated addition of kinetic energy that has been demonstrated by experiment (Kuhl, et. al. 1998), without explicitly solving the chemistry, or resolving the mesh to capture small-scale vorticity.

This effort is intended to complement the existing capability of either coupling ALE3D blast simulations with DYNA3D or performing fully coupled ALE3D simulations to predict building or component failure, for applications in National Security offensive strike planning as well as Homeland Defense infrastructure protection.
\end{abstract}

\section{INTRODUCTION AND BACKGROUND}

When an explosive detonates, a shock and a fireball are produced. The detonation products in the expanding fireball contain combustible hydrocarbons, such as $\mathrm{CO}$ and $\mathrm{C}$, that react at high temperatures with oxygen in the air. The combustion of these products releases energy into the fireball as it expands. The combustion equivalently increases the total energy of the detonation and its contribution is referred to as afterburn energy. 
Afterburn is measured experimental by measuring the overpressure in a fixed location after an explosive is detonated in a closed system (Kuhl, et. al.1998). Time histories of the pressure in a sealed container initially filled with 1 bar of air show this effect when the overpressure in the tank stabilizes above zero for a long duration after the detonation, while the exact same charge and tank filled with nitrogen asymptotes to atmospheric pressure. This is a direct result of the addition of combustion energy from afterburn and, in this experiment, the overpressure for the air case stabilizes between 2.5 and 3 bar (Figure 1). This combustion energy is not negligible for blasts in an enclosure, and it accounts for roughly $70 \%$ of the total energy found in an explosion. For example, the heat of detonation, $\Delta \mathrm{H}_{\mathrm{d}}$, for TNT alone is $-247 \mathrm{kcal} / \mathrm{mol} \mathrm{TNT}$, while the $\Delta \mathrm{H}$ from afterburn is $-567 \mathrm{kcal} / \mathrm{g} \mathrm{mol} \mathrm{TNT}$, so the total energy is $-814 \mathrm{kcal} / \mathrm{mol}$ TNT (Figure 2).

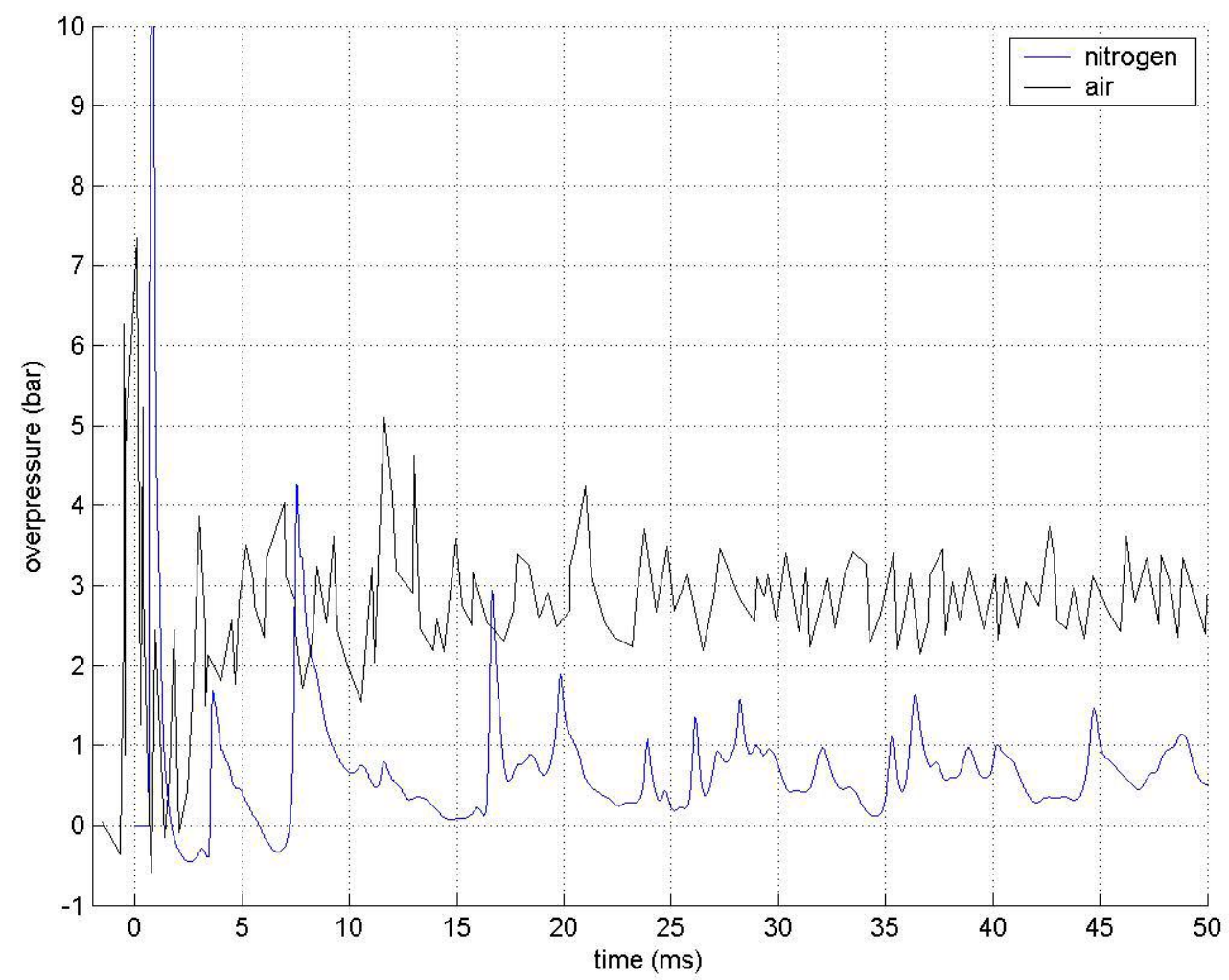

Figure 1: Comparison of confined explosions in air and $\mathrm{N}_{2}$ environments 


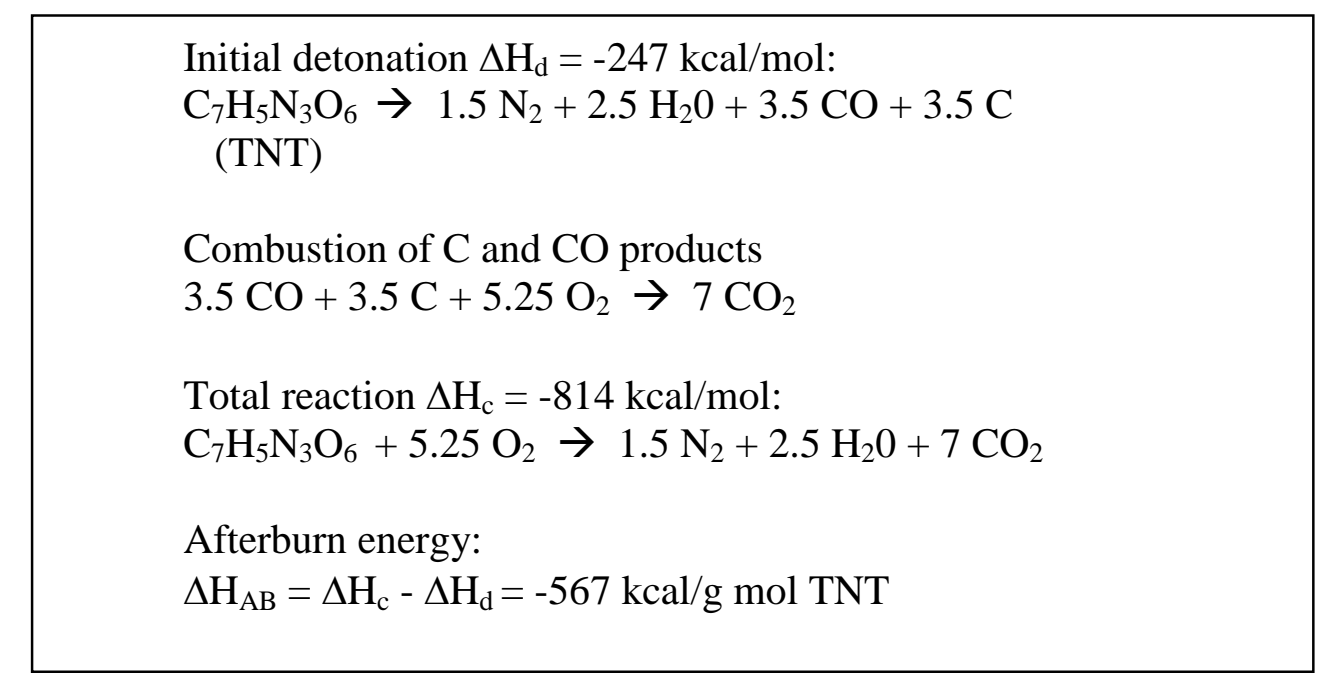

Figure 2: Chemistry of reaction from Cooper (1996) pp 132-3

The goal of this study is to characterize the source term for structural defeat or component defeat, and it is imperative that the energies from the blast are calculated appropriately. Consequently, this paper documents the development of an empirical energy deposition model that enhances the energy deposition into the explosion products in ALE3D.

Work similar to this has been proceeding in A-Division at LLNL for years and they are implementing a 3-D Adaptive Mesh Refinement (AMR) code as their tool to explicitly simulate the chemistry of the problem. Their research has shown that small-scale vorticity is what drives the mixing and subsequent afterburn combustion. Although the AMR code does an excellent job on afterburn predictions and turbulent mixing of detonation products in enclosed geometries, resolving small-scale vorticity is a computationally demanding problem. For national security and military applications of interest, there is a need for a more empirical, "quick and dirty" analysis methodology that can be performed at relatively coarser mesh resolutions. 


\section{Procedure}

Using the aforementioned data from the HEAF tests, a half symmetry finite element mesh of a sealed pressure vessel is built in True Grid for analysis in ALE3D. The model includes a single $800 \mathrm{~g}$ cylindrical TNT charge, surrounded by a mesh of air, and enclosed by a stainless steel vessel (Figure 3).

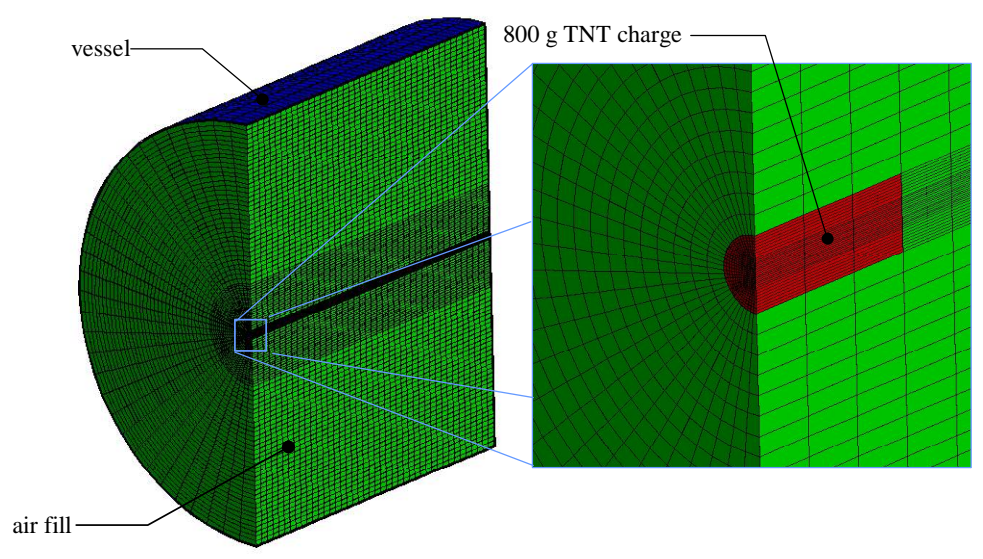

Figure 3: Graphic of ALE3D problem

It is assumed that there is zero energy loss to the walls (the time scale is too short for heat transfer) and that gravity does not act on the expanding products. The JonesWilkins-Lee (JWL) equation on state (Dube, et. al., 1999) is used to calculate the expansion of the detonation products. JWL takes the energy of detonation as input and has been shown to reproduce the correct energy and far-field pressure time histories compared against experiments. It will not, however reproduce the near-field pressure wave, but JWL was chosen here over a programmed burn model because the volumetric burn approach of JWL is less computationally draining, and our concern here is only farfield pressure. There was also a tritonal (TNT $+20 \%$ aluminum powder) case study in the referenced experiment, but that was not looked at here because the effects of 
afterburning detonation products is masked (and enhanced) by the burning of the entrained aluminum powders.

The JWL alone will only predict the oxygen-deprived environment because it does not account for afterburn. For the afterburn phenomenology, energy is added directly into the explosive products to create a time-dependent-JWL. The JWL block is set up as it would be for a simple TNT volumetric burn. Parameters used are (in "B-Division" units) $\rho=1.632 \mathrm{~g} / \mathrm{cc}$, Eo = 0.078 Mbar, $\mathrm{A}=4.5138 \mathrm{Mbar}, \mathrm{B}=0.105784 \mathrm{Mbar}, \mathrm{R}_{1}=4.5, \mathrm{R}_{2}=$ 1.5 , and $\omega=0.25$ (Souers, 1999). The magnitude of the energy deposition comes from the CHEETAH detonation state code that predicts the final thermodynamic state of an explosive and it includes detonation as well as afterburn energy, however it does not give burn rate. For this $800 \mathrm{~g}$ TNT example, the afterburn energy is calculated by C. Souers of Chemistry and Chemical Engineering Division to be $4826.5 \mathrm{~kJ} / \mathrm{mol}$ TNT (Souers, 2000). Initial detonation and the subsequent afterburn occur over a period of time, it is inaccurate to add all the energy into the calculation at once, so a time dependency must be derived.

\section{DERIVING THE ENERGY DEPOSITION}

As previously stated, the total afterburn energy is given by the CHEETAH detonation state code. The hypothesis here is that this energy is deposited into the explosive products as the fireball expands and is completed by the time the fireball reaches its maximum. This methodology then predicts that the bulk of the combustion occurs as the fireball is expanding. Recall that a fireball expands with the shock, reaches a maximum radius, then contracts while the shock continues on. For simple geometries and open air explosions of TNT, the fireball radius can be calculated from tables and the time of influence is calculated by the detonation velocity. For more complicated geometries a JWL-non-afterburn expansion scenario can be calculated to predict the fireball expansion. Then, the energy from CHEETAH is added from early time to the time that the fireball stagnates. Although cumbersome, because the calculations are done twice 
(once without energy and then again with the energy deposition), this seems to best reproduce the experimental data while still being physical.

For this problem the author also looked using the mass fraction of burned detonation products as a function of time as a multiplier for the afterburn energy and also having ALE3D explicitly calculated vorticity in the explosive products, but neither of these situations gave realistic answers.

For this case, an ALE3D calculation was performed without afterburn that tracked the fireball expansion and showed the maximum fireball radius of $160 \mathrm{~cm}$ at $\sim 3.5 \mathrm{~ms}$. The afterburn energy from CHEETAH is $4826.5 \mathrm{~kJ} / \mathrm{mol}$ TNT and the molecular weight of TNT is $227.13 \mathrm{~g} / \mathrm{mol}$. Energy divided by molecular weight yields $21.25 \mathrm{~kJ} / \mathrm{g}$ TNT or $0.2125 \mathrm{Mb} \mathrm{cc/g}$ in B-Division units (for input to ALE3D). Starting the deposition at 0.1 ms to allow for the initial detonation expansion transient and going out to the maximum fireball radius at $3.5 \mathrm{~ms}$ gives a mass energy rate of $6.25 \mathrm{e}-5 \mathrm{Mb} \mathrm{cc} / \mu \mathrm{s}$ for a constant deposition and $1.25 \mathrm{e}-46.25 \mathrm{e}-5 \mathrm{Mb} \mathrm{cc} / \mu \mathrm{s} g$ for a linear deposition over the range of fireball expansion. Figure 4 shows the results of these calculations. The constant deposition had an average value of 3.0 bar and the linear deposition had an average of 2.7 bar - both within the 2.5-3 bar range shown in experiment. 


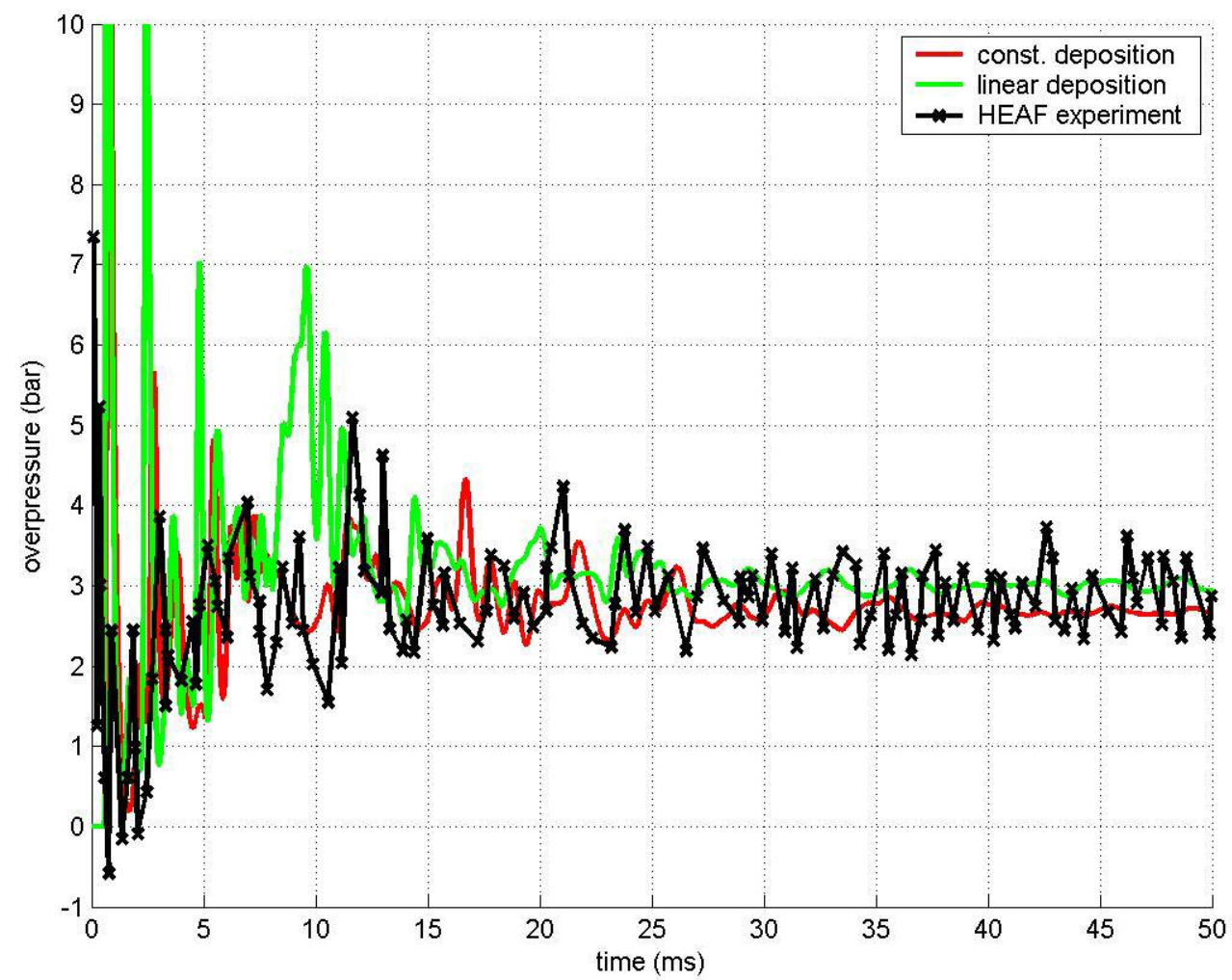

Figure 4: Comparison of overpressure time histories with experimental data

\section{CONCLUSION}

Presented here is an empirical resource to produce afterburn phenomenology in an ALE3D simulation. This methodology derived a simple to implement, and physically based energy deposition term that produces realistic answers based on comparison with experiment. Users should exercise caution in the implementation of this methodology that has only been tested on one experiment and timescale. 


\section{REFERENCES}

[1] Cooper, P.W., Explosives Engineering, Wiley-VCH: New York, 1996.

[2] Dube, E., et. al., "Users Manual for ALE3D: An Arbitrary Lagrange/Eulerian 3D Code System”, version 2.8.6, October 25, 1999.

[3] Kuhl, A. L., et. al, "Confined Combustion of TNT Explosion Products in Air", $8^{\text {th }}$ International Colloquium on Dust Explosions, UCRL-JC-131748, August 31, 1998.

[4] Souers, C., personal correspondence, December 1999 and March 2000. 